\section{Names from all nations}

SIR - As one responsible for the United Nations University abstract journal, largely aimed at readers in developing countries, I am not surprised that your readers' interest in Chinese names has now extended to South Indian names (Nature 17 February, p.560). As both an editor and UN employee, I can see a need for an international guide to personal names.

For Chinese names, we have had to compile our own guide, but the problem of identifying surnames extends to many more cultures than those of China and South India. The problem is complicated by the fact that surnames are not always those used in formal address.

Having assumed that by surname we mean family name, it is helpful to use the term formal name for that name or shortest string of names that can be used following a title such as Dr. For Henry Kissinger and Sun Yat-sen, the surnames are formal names and we may address them formally as Dr Kissinger and Dr Sun. This is important since what we are in fact looking for are formal names, which are usually but not always surnames. Instances where we have encountered this divergence will be identified among the following sample list of customs regarding the use of surnames.

Spanish: Typically three or more names with the last two as surnames, sometimes connected by " $y$ ", although the second surname is often dropped or abbreviated. The formal name begins with first surname and would include second surname in very formal usage.

Icelandic: Usually two names but no surname. First is formal name in Iceland but second accepted as formal internationally.

Hungarian: Traditionally two names with surname-formal name first but second accepted as formal internationally.

Arabic: Highly variable, often with many names. Formal name often consists of two or three names including articles which can be joined together.

Burmese: One, two or three names typical, but no surname. All names included in formal name.

Thai: Usually two names with surname last but formal name first.

Vietnamese: Usually two or three names. First is surname-formal name.

Japanese: Two names with surnameformal name first when rendered in Japanese but almost always reversed in non-East Asian languages or scripts.

Korean: Usually three names with surname-formal name first. First is sometimes placed last in non-East Asian situations. Same as Chinese usage.

Indonesian: Great variety due to mosaic of ethnic, religious and class groups. For Javanese sometimes one name for all purposes. For some groups including Sundanese, two names often used with first as formal name. Very complex.

African: Great variety due to mosaic of ethnic, religious and colonial traditions In Rwanda, typically two names with surname last but formal name first.

This sample is far from exhaustive but should give an idea of the diversity involved. Mention could also be made of the problems of identifying titles which are often attached to names, as in the case of $\mathrm{U}$ Thant where $U$ means roughly uncle.

Perhaps an international effort to produce a definitive guide would go a long way towards saving much embarrassment in the future. The very diversity of names reflects the fact that human institutions are also very diverse, reflecting the mental constructions that societies have developed to handle the problem of naming their members. We can only hope that the computer forms M.V. Ramana describes will not force all this diversity into monotonous uniformity.

United Nations University, WAL.TER SHEARER

Tokyo, Japan

\section{West is east?}

SIR - The Greenwich meridian fairly neatly divides the Antarctic continent into an eastern half and a western half. It is in this manner that the place-names "East Antarctica" and "West Antarctica" have come about. Logical as these names seem at first sight, there is no doubt they do give rise to ambiguities, for example "East Antarctica" is to the westward for Australians and New Zealanders; nor does the meridian follow the geographical dividing line of the Transantarctic Mountains and the east side of the Filchner Ice Shelf. The problem is highlighted by Dennis E. Hayes at the end of an introduction to a report ${ }^{1}$ of the Deep Sea Drilling Project of Glomar Challenger, 1972-73, when this research vessel was working in the Southern Ocean:

"In parts of East Antarctica West Antarctica is east, in others west. This of course depends on if you are in the east East Antarctica or west. However, if you are in west West Antarctica, East Antarctica is west unless you want to go to west East Antarctica in which case it is east. The same holds for east West Antarctica only in the reverse except that if you ivant to go to west East Antarctica, you still go east. No wonder we don't know what we found!"

May I therefore draw the attention of your readers and contributors ${ }^{2}$ to the alternatives of "Greater Antarctica" and "Lesser Antarctica", which in addition to being more fitting from the geographical aspect are also more easily remembered. They have been defined as follows ${ }^{3}$ :

Greater Antarctica: A general name for the major region of Antarctica lying in the section of the Indian Ocean side of the Transantarctic Mountains.

Lesser Antarctica: A general name for the minor region of Antarctica lying on the Pacific Ocean side of the Transantarctic Mountains. Lesser Antarctica includes the Antarctic Peninsula.
These definitions were adopted in 1960 by the UK Antarctic Place-names Committee, by the Antarctic Names and Medal Committee of Australia and by the New Zealand Antarctic Place Names Committee. The US Advisory Committee on Antarctic Names approved the names East Antarctica and West Antarctica in 1962, which it felt to be firmly established among scientists and others. The late Dr Brian Roberts, one of the leading architects of the Antarctic Treaty, believed that with time a consensus would evolve in the light of common usage by the scientists and other workers in this field.

ANN SAvours

Old Royal Observatory, National Maritime Museum,

London SE10, UK

1. Haycs, D.E. Init. Rep. DSDP 28, I (1975).

2. Nature 301, 551-552 (1983)

3. Roberts, B.B. Br. Antarctic Survey Bull. 53, 257-260 (1981).

\section{Is anybody there?}

SiR - I am pleased that the search for extraterrestrial intelligence (SETI) is beginning again in earnest. The current effort by Harvard, Stanford, Berkeley and the National Aeronautics and Space Administration is exciting. It is logical to search in the microwave region near the hydrogen fine-structure wavelength of 21 $\mathrm{cm}$ since the phenomenon leading to this emission will be known by technologically oriented societies.

But I would like to make a complementary SETI proposal. I suggest that a search for extraterrestrial signals be made at extremely low electromagnetic frequencies in the neighbourhood of $10 \mathrm{~Hz}$.

There is a logical basis for this suggestion. The Earth, with its ionosphere, forms a natural detector of electromagnetic radiation. If the Earth-ionospheric system is considered as a conducting sphere surrounded by a conducting shell hundreds of kilometres from the surface of the Earth, it becomes a cavity resonator. One can then calculate the eigen-modes of this system'. The first few eigenstates occur roughly at 8,14 and $20 \mathrm{~Hz}$.

This matter is familiar to atmospheric scientists. Most of the signals observed at these frequencies are generated by lightning bolts around the Earth.

A comparison of the microwave and extremely low frequency approaches to SETI points out relative advantages and disadvantages. The microwave scenario leads to highly directional beams which are appropriate if you know where you want to send or receive. On the other hand, extremely low frequency transmissions are by necessity rather isotropic (like a dipole radiator) and can therefore be sent or received fairly universally at a substantial cost in intensity.

FRANK J. LOEFFLER

Department of Physics,

Purdue University,

West Lafayett, Indiana, USA

1. Schumann, W.O. Z. Nuturf. 7a, 149-154; 250-252 (1952). 